Med Klin Intensivmed Notfmed 2016 111:668-669 DOI 10.1007/s00063-016-0231-3

Online publiziert: 2. November 2016

๑) Springer-Verlag Berlin Heidelberg 2016

CrossMark

\author{
H.-R. Arntz ${ }^{1}$ S. Wolfrum ${ }^{2}$ \\ ${ }^{1}$ Abteilung für Kardiologie und Pulmologie, Charité - Universitätsmedizin Berlin, Campus Benjamin \\ Franklin, Berlin, Deutschland \\ ${ }^{2}$ Interdisziplinäre Notaufnahme, Universitätsklinikum Schleswig Holstein, Campus Lübeck, Lübeck, \\ Deutschland
}

\title{
Kardiopulmonale Wiederbelebung
}

hat bisher wenig Einfluss gezeigt - mit regionalen Unterschieden. Zumindest teilweise mag die geringe Quote der Laienreanimation dadurch erklärt sein, dass sich 70-80\% der plötzlichen Herztodesfälle in häuslicher Umgebung ereignen und Angehörige in dieser Situation aus persönlicher Betroffenheit überfordert sind, rational zu handeln. Entsprechend sind die Ersthelferquoten in häuslicher Umgebung besonders gering und die Überlebenschancen schlechter als bei Ereignis in der Öffentlichkeit. Dies nur als ein Hinweis auf die unverändert bestehende dringliche Notwendigkeit der Forschung selbst an scheinbar einfachen Problemen.

Es ist leicht nachvollziehbar, wie viel schwieriger es ist, Forschung durchzuführen und möglichen Fortschritt nachzuweisen bei wesentlich komplexeren Problemen der Reanimatologie. Unter anderem das breite Spektrum der Ursachen des Kreislaufstillstands, die individuell unterschiedlichen und anderen äußeren Bedingungen, Alter und Geschlecht der Betroffenen und der möglichen Helfer, Vorerkrankungen der Patienten, das Spektrum der Möglichkeiten des Rettungsdienstes, Nachbehandlung und eine Vielzahl weiterer Faktoren bilden ein nur schwer entwirrbares Geflecht unterschiedlichster Einflüsse auf das Ergebnis eines CPRVersuchs. Gleichzeitig ist das breite Spektrum an Einflüssen ein grundsätzliches, vor allem auch statistisches Problem, da für den Menschen unmittelbar relevante Aussagen zu Methoden und Maßnahmen meist nur mithilfe relativ großer
Patientenzahlen gewonnen werden können.

Die sowohl als Notärzte als auch Kliniker erfahrenen Autoren der vorliegenden Beiträge zur kardiopulmonalen Reanimation haben sich deshalb darum bemüht, einige aktuelle Fragen auf dem Gebiet der Wiederbelebung anzusprechen, ohne den Anspruch zu stellen, endgültige Antworten zu haben.

Die Freiburger Gruppe stellt die grundsätzlichen Regeln der CPR beim Erwachsenen dar und dies nicht, ohne einige grundsätzlich ungelöste Probleme im Rahmen dieser Regeln anzusprechen. Stichworte sind hier z. B. die Rolle von Adrenalin, das Atemwegsmanagement oder auch die therapeutische Hypothermie.

C. Mochmann von der Charité setzt sich aus kardiologischer Sicht mit der Nachbehandlung nach zunächst erfolgreicher kardiopulmonaler Reanimation auseinander. Eine besondere Rolle kommt aus dieser Sicht der Diagnostik, der Indikationsstellung, den Möglichkeiten und den ungelösten Fragen der akuten Koronarintervention zu. Die besondere Bedeutung dieser Fragestellung wird mit Blick auf die Tatsache klar, dass die Mehrzahl der Kreislaufstillstände auf dem Hintergrund akuter Manifestationen oder akuter Verschlimmerungen einer koronaren Herzkrankheit entstehen. Probleme sind noch fehlende Ergebnisse randomisierter Untersuchungen auf der einen Seite und die Notwendigkeit der Verbringung der Patienten über u. U. große Entfernungen in ein Interventionszentrum. 
Der Beitrag aus Wien beleuchtet kritisch die Möglichkeiten technischer Unterstützungssysteme bei der CPR. Sie sind möglich als „Bridging“-Instrumente für Patienten, bei denen unter Reanimationsbedingungen ein Transport, z. B. von der Intensivstation zur Koronarintervention, bewerkstelligt werden muss. Der Routineeinsatz verschiedener mechanischer Kompressionshilfen hat dagegen bei der prähospitalen Reanimation die Erwartungen nicht erfüllt ebenso wenig wie der Einsatz von Hilfsmitten zur Thoraxkompression in Kombination mit speziellen Zusatzventilen am Tubus, die den pulmonalen Blutrückfluss bei der Beatmung verstärken. Komplette Herz-Lungen Unterstützungssysteme sind die fortgeschrittenste Entwicklung auf diesem Gebiet. Ihr Einsatz - vor allem wiederum zum „Bridging“ - ist allerdings zunächst auf hoch spezialisierte Zentren begrenzt, bildet aber den Ansatz zu völlig neuen Perspektiven für längere Transporte in ein Interventionszentrum und möglicherweise sogar für die prähospitale CPR.

BA Leidel und KG Kanz (Berlin/ München) geben eine Übersicht über das komplexe Problem der CPR nach Trauma. Traditionell besteht eine Neigung eine Reanimation nach Trauma als sinnlos zu betrachten. In ihrer Übersicht weisen Leidel und Kanz nachdrücklich darauf hin, dass im Gegensatz zu dieser verbreiteten Meinung auch in dieser verzweifelten Situation Möglichkeiten zu gezieltem lebensrettendem Vorgehen bestehen. Sicher ist einiges davon dem sehr erfahrenen Notarzt vorbehalten oder erst unter Klinikbedingungen realisierbar. Dennoch sollten diese Möglichkeiten Anlass sein, dem Fatalismus bei traumatischem Kreislaufstillstand keinen Raum zu geben.

Wir hoffen, Ihnen mit diesen Beiträgen zur Wiederbelebung in Ausgabe
8/2016 der Zeitschrift Medizinische Klinik-Intensivmedizin und Notfallmedizin einige Einblicke in aktuelle Fragestellungen zum Stand der Reanimatologie und Anregungen für Ihre tägliche Arbeit zu geben.

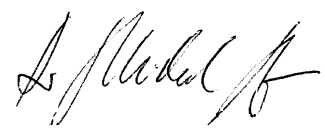

Prof. Dr. H.-R. Arntz

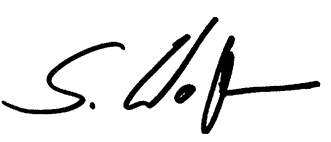

Dr. S. Wolfrum

\section{Korrespondenzadresse}

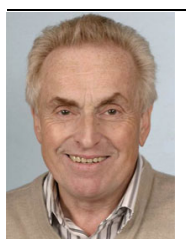

Prof. Dr. H.-R. Arntz

Abteilung für Kardiologie und Pulmologie, Charité Universitätsmedizin Berlin, Campus Benjamin Franklin Hindenburgdamm 30, 12200 Berlin, Deutschland HRArntz@t-online.de

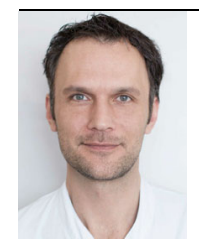

\section{Dr. S. Wolfrum}

Interdisziplinäre Notaufnahme, Universitätsklinikum Schleswig Holstein, Campus Lübeck

Ratzeburger Allee 160, 23538 Lübeck, Deutschland Sebastian.Wolfrum@uksh.de

Interessenkonflikt. H.-R. Arntzund S. Wolfrumgeben an, dass kein Interessenkonflikt besteht.
Notfallversorgung von Patienten mit alkoholbedingten Erkrankungen

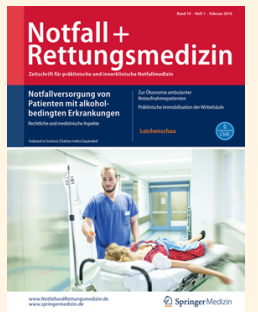

Alkoholisierte Patienten im Rettungsdienst sind oft nur schwer einschätzbar, da die Alkoholmenge und der Zeitpunkt der Einnahme meist unbekannt sind, die Anamnese und auch die körperliche Untersuchung Mischintoxikationen ergeben sowie Begleitverletzungen vorliegen können.

Lesen Sie in der Ausgabe 1/2016 von Notfall+Rettungsmedizin mehr über die rechtlichen Besonderheiten in der präklinischen Versorgung, die Beurteilung der Einsichtsfähigkeit, den Umgang mit aggressiven $\mathrm{Pa}$ tienten und die spezielle rechtliche Situation bei der Anordnung von Blutalkoholentnahmen. Zudem werden die Epidemiologie und Pathophysiologie der akuten Alkoholintoxikation, medizinische Therapiestandards in Notaufnahmen, sowie Komplikationen und Alkoholfolgeerkrankungen und wesentliche Differenzialdiagnosen thematisiert.

Lesen Sie im Schwerpunktheft mehr zu folgenden Themen:

- Besonderheiten der präklinischen Versorgung alkoholisierter Patienten

- Anordnung von Blutalkoholentnahmen durch die Polizei im Rettungsdienst

- Akute Alkoholintoxikation. Vorgehen bei Erwachsenen und Jugendlichen

- Alkoholentzugsdelir und akute Komplikationen

- Alkoholpatienten als Risikopatienten. Was muss der Notfallmediziner beachten?

Bestellen Sie diese Ausgabe zum Preis von 39,- EUR zzgl. Versandkosten bei Springer Customer Service Center, Kundenservice Zeitschriften

Tel.: +49 6221-345-4303

E-Mail: leserservice@springer.com 